\title{
14.6-GHz $\mathrm{LiNbO}_{3}$ Microdisk Photonic Self-Homodyne RF Receiver
}

\author{
Mani Hossein-Zadeh, Member, IEEE, and Anthony F. J. Levi
}

\begin{abstract}
Nonlinear optical modulation combined with simultaneous photonic and $\mathrm{RF}$ resonance in an $\mathrm{LiNbO}_{3}$ microdisk modulator is used to create a self-homodyne photonic $\mathrm{RF}$ receiver. Carrier and sidebands are mixed in the optical domain, and the modulated optical signal is detected using a photodetector. The photodetector has a bandwidth matched to the baseband signal. It filters out the high-frequency components and generates the baseband photocurrent. Receiver operation is demonstrated by demodulating up to $100-\mathrm{Mb} / \mathrm{s}$ digital data from a 14.6-GHz carrier frequency without any high-speed electronic components. A bit error rate of $10^{-9}$ is measured for $10-\mathrm{Mb} / \mathrm{s}$ downconverted digital data at $-15-\mathrm{dBm}$ received $\mathrm{RF}$ power. Preliminary results of employing this photonic RF receiver in a short-distance $K u$-band wireless link demonstrate the potential of using high-quality optical microresonators in $\mathrm{RF}$ receiver applications.
\end{abstract}

Index Terms-Electrooptic modulation, $\mathrm{LiNbO}_{3}$ microdisk modulator, microwave receiver, nonlinear modulation.

\section{INTRODUCTION}

$\mathbf{P}$ ROCESSING microwave and millimeter-wave signals in the optical domain has been the subject of research for the past few years [1]-[3]. Placing a RF signal on an optical carrier enables a wide variety of photonic signal processing techniques and, at the same time, avoids the use of lossy transmission lines and high-speed electronic devices.

One of the key operations in microwave communication is frequency mixing. Several techniques have been suggested for $\mathrm{RF}$ mixing in the optical domain, such as nonlinear modulation in a Mach-Zehnder (MZ) modulator [4], [5] and nonlinear detection in a photodiode [6]. This paper introduces a new photonic RF mixing technique that exploits simultaneous RF and optical resonance in an electrooptic microdisk resonator. This optical mixer is used to realize a photonic $\mathrm{RF}$ receiver, which does not incorporate any high-speed electronic components.

In a conventional super-heterodyne RF receiver architecture, a local oscillator (LO) and mixer are used to down-convert the signal to IF frequencies. Baseband information is subsequently extracted from the IF signal in a detector/demodulator. Alternatively, in a direct-conversion (homodyne) radio receiver, baseband information is obtained by mixing the received signal

Manuscript received April 1, 2005; revised August 9, 2005. This work was supported in part by the National Aeronautics and Space Administration and by the Defense Advanced Research Projects Agency.

M. Hossein-Zadeh was with the Department of Electrical Engineering, University of Southern California, Los Angeles, CA 90089 USA. He is now with the Vahala Research Group, California Institute of Technology, Pasadena, CA 91125 USA (e-mail: mhz@caltech.edu).

A. F. J. Levi is with the Department of Electrical Engineering, University of Southern California, Los Angeles, CA 90089 USA (e-mail: alevi@usc.edu).

Digital Object Identifier 10.1109/TMTT.2005.863061 and the LO without using an IF frequency [7]. In addition to such approaches, self-heterodyne techniques have been proposed to reduce the number of components as well as size, weight, and power consumption in high-carrier frequency (millimeter wave), short-distance applications [8]. In a self-heterodyne transmission system, the transmitter broadcasts an RF modulated signal and the local carrier so the IF signal can be down-converted by mixing the received carrier and modulated signal in a nonlinear device called a self-mixer. Although such an approach suffers from reduced power efficiency, it can lower overall cost and complexity in millimeter-wave local area networks (LANs) and indoor wireless transmission systems [8].

The simplest RF receiver is a video receiver that combines direct-conversion and self-mixing techniques to down-convert the information from a high-frequency carrier without an LO. The self-mixing is performed in a diode (e.g., Schottky) operating in the square-law region (typically from noise level to about $-20-\mathrm{dBm}$ input power). We refer to this down-conversion method as self-homodyne. A video receiver is typically 35-40 dB less sensitive than a heterodyne receiver; nevertheless, its simplicity and low cost outweigh this disadvantage for certain applications.

The proposed photonic self-homodyne RF receiver replaces the function of a single-ended diode or field-effect transistor (FET) mixer in a transmitted carrier wireless link, with a sensitive optical modulator that performs self-mixing of the carrier and the sidebands in the optical domain. We show that the second-order nonlinearity in the transfer function of an $\mathrm{LiNbO}_{3}$ microdisk optical modulator when biased at its minimum transmission point may be used to realize the square-law functionality. Receiver operation is demonstrated experimentally by demodulating a digital data from a 14.6-GHz RF carrier frequency.

\section{Self-Homodyne Photonic RF Receiver}

In a microdisk photonic mixer, the nonlinear dependence of the microdisk modulator's transmitted optical power $\left(P_{\mathrm{ot}}\right)$ on applied RF voltage $\left(V_{\mathrm{RF}}\right)$ is the source of nonlinearity in the system. In the resonant cavity of our microdisk, modulation of optical phase by the linear electrooptic (Pockels) effect results in nonlinear modulation of optical intensity. The strength of this nonlinearity depends on the laser wavelength offset from resonance. Fig. 1 illustrates the photonic self-homodyne RF receiver architecture. The received RF signal contains both sidebands and the center frequency (transmitted-carrier double-sideband modulation format) and is fed to a microdisk optical modulator biased at its optimally nonlinear operating point. The carrier and sidebands are mixed through the second-order nonlinearity $\left(P_{\mathrm{ot}} \propto V_{\mathrm{RF}}^{2}\right)$, hence the optical output intensity spectrum 


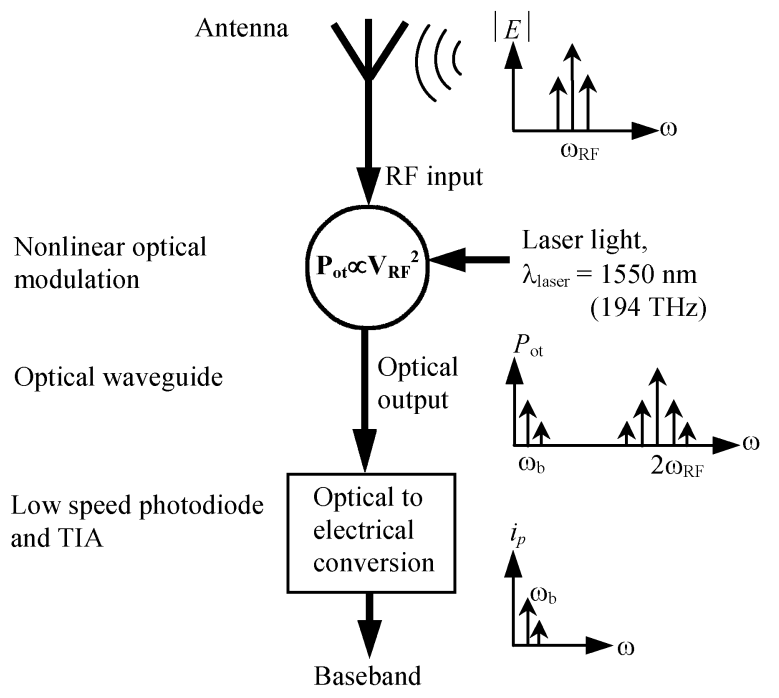

Fig. 1. Schematic diagram of the photonic self-homodyne RF receiver. The transmitted carrier RF signal is received by the antenna and is directly fed to a square-law optical intensity modulator. Through nonlinear optical modulation, the optical output intensity spectrum contains the baseband and high-frequency components that are filtered out by the response of the low-speed photodetector.

contains the baseband and high-frequency products around the second-harmonic of the carrier frequency. A photoreceiver with a bandwidth matched to the baseband signal generates the baseband photocurrent $\left(i_{\mathrm{p}}\right)$ and automatically filters out the highfrequency components. The bandwidth of any electronic circuitry used in the system is no greater than that of the baseband signal.

The electrooptic transfer function of an optical intensity modulator $P_{\mathrm{ot}}\left(V_{\mathrm{RF}}\right)$ can be expanded around $V_{\mathrm{RF}}=0$ to give

$$
\begin{aligned}
P_{\mathrm{ot}} & =P_{o}^{(0)}+P_{o}^{(1)}+P_{o}^{(2)}+\ldots \\
& =N_{0}+N_{1} V_{\mathrm{RF}}+\frac{N_{2}}{2} V_{\mathrm{RF}}^{2}+\ldots
\end{aligned}
$$

Here, $N_{i}(i>0)$ is the $i$ th derivative of $P_{\mathrm{ot}}\left(V_{\mathrm{RF}}\right)$ at $V_{\mathrm{RF}}=0$ and $N_{0}$ is the transmitted optical power at $V_{\mathrm{RF}}=0$. At a fixed wavelength, the magnitude of $N_{i}$ depends on modulator properties and the chosen bias point. The first-order term in (1) generates linear optical intensity modulation $\left(P_{\mathrm{ot}} \propto V_{\mathrm{RF}}\right)$ while other terms contribute nonlinear frequency components. Usually, such nonlinearities are minimized in conventional direct-detection optical communication links. If the RF voltage amplitude is sufficiently small and the modulator is biased at its extreme transmission point (where $d P_{\mathrm{ot}} / d V_{\mathrm{RF}}=0$ ), the second-order term $\left(P_{o}^{(2)}\right)$ dominates the behavior of the modulator, and the transmitted optical power $\left(P_{\mathrm{ot}}\right)$ dependence on voltage around $V_{\mathrm{RF}}=0$ will be similar to an ideal square-law mixer with

$$
P_{\mathrm{ot}} \approx N_{0}+\frac{N_{2}}{2} V_{\mathrm{RF}}^{2} .
$$

If the baseband is a pure sinusoidal signal, the received RF voltage can be written as

$$
V_{\mathrm{RF}}=V_{0}\left[1+m \cos \left(\omega_{\mathrm{b}} t\right)\right] \cos \left(\omega_{\mathrm{RF}} t\right)
$$

where $m$ is the RF modulation index, $\omega_{b}$ is the baseband frequency, and $\omega_{\mathrm{RF}}=2 \pi f_{\mathrm{RF}}$ is the RF carrier frequency. The second-order term can be written as

$$
\begin{aligned}
P_{o}^{(2)} & =\frac{N_{2}}{2} \times V_{\mathrm{RF}}^{2} \\
& =\frac{N_{2}}{2} V_{0}^{2}\left[1+m \cos \left(\omega_{\mathrm{b}} t\right)\right]^{2} \cos ^{2}\left(\omega_{\mathrm{RF}} t\right) .
\end{aligned}
$$

Expanding the right-hand side of (4), one obtains a dc term equal to $\left(N_{2} V_{0}^{2} / 4\right)\left(1+m^{2} / 2\right)$ high-frequency components centered around $2 \omega_{\mathrm{RF}}$ given by

$$
\begin{aligned}
\frac{N_{2} V_{0}^{2}}{4}[ & \left(1+m^{2} / 2\right) \cos \left(2 \omega_{\mathrm{RF}} t\right) \\
& +\left(m^{2} / 2\right) \cos \left(2 \omega_{\mathrm{b}} t\right) \cos \left(2 \omega_{\mathrm{RF}} t\right) \\
& \left.+2 m \cos \left(\omega_{\mathrm{b}} t\right) \cos \left(2 \omega_{\mathrm{RF}} t\right)\right]
\end{aligned}
$$

and the two down-converted low-frequency terms at $\omega_{b}$ and $2 \omega_{b}$ are given by

$$
\frac{N_{2} V_{0}^{2} m^{2}}{8} \cos \left(2 \omega_{\mathrm{b}} t\right)+\frac{N_{2} V_{0}^{2}}{2} m \cos \left(\omega_{\mathrm{b}} t\right) .
$$

The total second-order modulated optical power is

$$
P_{o, \max }^{(2)}=\left(1+m^{2}+2 m\right) \frac{N_{2}}{2} V_{0}^{2}
$$

which is the maximum amplitude in (4).

The amplitude of the baseband modulated optical power is equal to $m N_{2} V_{0}^{2} / 2$, so the baseband photocurrent can be written as

$$
i_{\mathrm{p}}=R N_{2} \frac{V_{0}^{2}}{2} m \cos \left(\omega_{\mathrm{b}} t\right)+i_{\mathrm{DC}}
$$

where $R$ is responsivity of the photodiode (typically $0.8-0.9 \mathrm{~A} / \mathrm{W}$ ) and $i_{\mathrm{DC}}$ is the dc (time-independent) photocurrent. Usually, the photodiode is followed by a transimpedance amplifer (TIA) which acts as a current-to-voltage converter. In this case, the baseband voltage can be calculated by multiplying $i_{\mathrm{p}}$ [see (8)] and the transimpedance of the TIA. Notice that the bandwidth of the photodiode and the TIA are much less than $\omega_{\mathrm{RF}}$, so the high-frequency terms in (5) do not contribute an ac component to the photocurent.

The term $R N_{2}$ represents the combined response of the laser, microdisk modulator, and the photodiode as a square-law RF mixer. By way of comparison, if an electronic diode is used as a square-law mixer, then $R N_{2}$ in (8) is replaced by $\alpha G_{\mathrm{d}}$ where $\alpha$ is $e / n k_{\mathrm{B}} T$ (and $e$ is electron charge, $k_{\mathrm{B}}$ is Boltzman's constant, $T$ is temperature, and $n$ is the ideality factor) and $G_{\mathrm{d}}$ is the dynamic conductance of the diode [9].

Equation (8) shows that the sensitivity of a photonic RF mixer depends on the magnitude of the second-order nonlinearity (determined by the modulator sensitivity and the transfer function $\left.P_{\text {ot }}\right)$ and the photodetector response. Given that most wireless links require only a limited bandwidth around a high-frequency carrier, a microdisk resonant optical modulator [10] is a suitable choice for this application since it has a large $N_{2}$ coefficient when biased at a maximum or minimum optical transmission of a high- $Q$ whispering-gallery (WG) resonance (that ideally has a Lorentzian transfer function). 


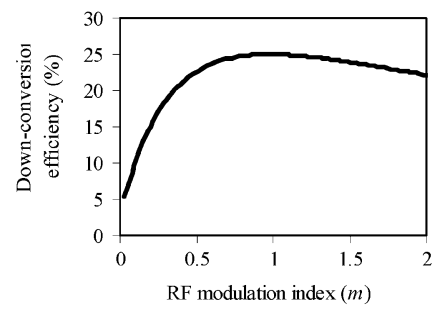

( a )

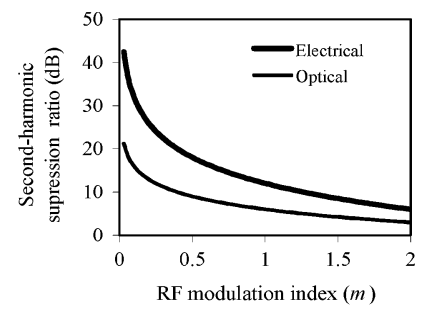

(b)
Fig. 2. (a) Calculated down-conversion efficiency $\left(P_{o}, \omega_{\mathrm{b}} / P_{o}^{(2)} \max \right)$ versus RF modulation index $(m)$. (b) Second-harmonic suppression ratio against $m$. The electrical and optical suppression ratios are related through: $P_{\mathrm{e}, \omega_{\mathrm{b}}} / P_{\mathrm{e}, 2 \omega_{\mathrm{b}}}=\left(i_{\omega_{\mathrm{b}}} / i_{2 \omega_{\mathrm{b}}}\right)^{2} \propto\left(P_{\mathrm{o}, \omega_{\mathrm{b}}} / P_{\mathrm{o}, 2 \omega_{\mathrm{b}}}\right)^{2}$

The efficiency of this down-conversion process may be defined as the ratio between the amplitude of the optical power modulated at $\omega_{b}$ and $P_{o, \max }^{(2)}$. This efficiency is limited by the generation of undesired frequency components at $2 \omega_{b}, 2 \omega_{\mathrm{RF}} \pm 2 \omega_{b}$, and $2 \omega_{\mathrm{RF}} \pm \omega_{b}$ as well as the dc component. The ratio of the optical power modulated at $\omega_{b}$ and $2 \omega_{b}\left(P_{o, \omega b} / P_{o, 2 \omega b}\right)$ is an indication of down-conversion linearity and is an important parameter in receiver operation. In the square-law regime, the strength of the second-order term in the expansion of a Lorentzian function dominates higher order terms, so the generation of higher harmonics of the baseband (e.g., $3 \omega_{\mathrm{b}}$ and $4 \omega_{\mathrm{b}}$ ) can be ignored. Equations (6) and (7) show that the down-conversion efficiency and the magnitude of $\left(P_{o, \omega \mathrm{b}} / P_{o, 2 \omega \mathrm{b}}\right)$ are determined by the RF modulation index $(m)$.

In Fig. 2, the down-conversion efficiency (a) and the secondharmonic suppression ratio (b) are calculated against $m$. The second-harmonic baseband term $\left(2 \omega_{\mathrm{b}}\right)$ can be suppressed relative to the baseband $\left(\omega_{\mathrm{b}}\right)$ by employing a transmitted carrier RF modulation format $(m<2)$, and the down-conversion efficiency reaches its maximum value of $25 \%$ around $m=1$. As may be seen at low modulation index, below 0.8 , down-conversion efficiency decreases while suppression ratio increases. Because of this inverse relation, the desired efficiency and suppression will determine an optimum modulation index for each application. Choosing $m=0.8$, an efficiency of about $25 \%$ and second-harmonic suppression of $7 \mathrm{~dB}$ optical ( $14 \mathrm{~dB}$ electrical, $\left.P_{\mathrm{e}} \propto i_{\mathrm{p}}^{2} \propto\left[P_{\mathrm{ot}}^{(2)}\right]^{2}\right)$ can be achieved.

\section{Microdisk OPTICAL MODULATOR}

\section{A. $\mathrm{LiNbO}_{3}$ Microdisk Modulator}

The optical resonator we use is a $z$-cut $\mathrm{LiNbO}_{3}$ microdisk that supports high- $Q>10^{6} \mathrm{WG}$ traveling-wave resonances [10].

We use a metal-ring RF resonator with the same diameter as the microdisk to modulate the optical modes in the $\mathrm{LiNbO}_{3}$ [11]. The RF resonator is placed on top of the $\mathrm{LiNbO}_{3}$ microdisk and is side coupled to a microstripline that is used to feed electromagnetic energy to the resonator. The fundamental RF resonant frequency $\left(f_{1}\right)$ of the loaded metal ring is designed to be equal to the optical free spectral range $\left(\Delta \nu_{\mathrm{FSR}}\right)$. Fig. 3(a) shows a photograph of the $\mathrm{LiNbO}_{3}$ microdisk modulator with diameter $D=3 \mathrm{~mm}$, thickness $h=0.4 \mathrm{~mm}$, and $\Delta \nu_{\mathrm{FSR}}=14.6 \mathrm{GHz}$.

The ring is magnetically coupled to the microstripline, and its coupling coefficient is tuned by adjusting the distance $g$

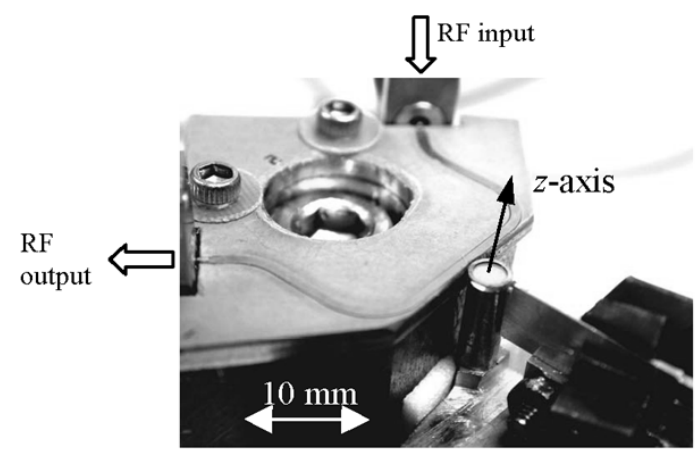

( a )

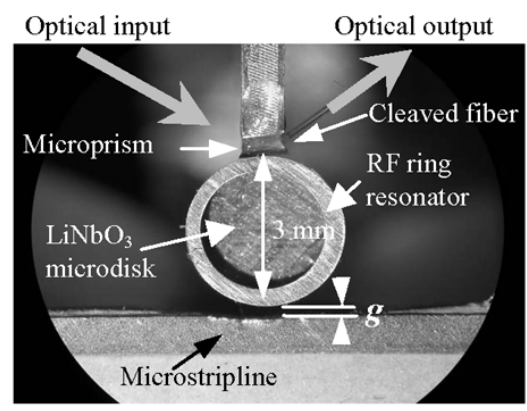

(b)

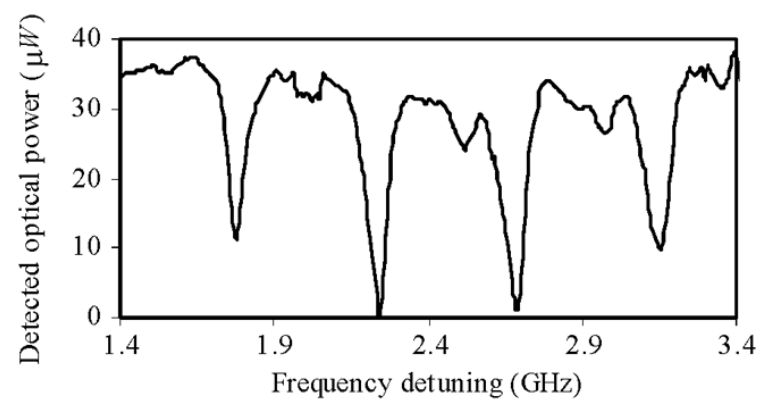

(c)

Fig. 3. (a) $\mathrm{LiNbO}_{3}$ microdisk modulator. (b) Close-up view of the modulator showing the microstripline, $\mathrm{LiNbO}_{3}$ microdisk, microprism, microring RF resonator, and the output fiber. (c) The typical measured TE-polarized optical transmission spectrum of the $\mathrm{LiNbO}_{3}$ microdisk. The frequency detuning is measured from a 194-THz $(1550 \mathrm{~nm})$ center frequency. The loaded $Q$ of these WG resonances is near $3 \times 10^{6}$.

between the microring and the microstripline. At resonance $\left(f_{\mathrm{RF}}=\Delta \nu_{\mathrm{FSR}}=f_{1}\right)$, the RF voltage amplitude seen by the optical mode is larger than the input voltage amplitude by a factor of $G_{\mathrm{V}}$ (which is the voltage gain factor) [11], [12]. We tune $g$ such that, at $f_{\mathrm{RF}}=\Delta \nu_{\mathrm{FSR}}$, the ring (RF resonator) is critically coupled to the microstripline, and, therefore, $E$-field intensity across the microdisk is maximized. Although in order to tune the ring resonator the RF output port is used to measure the throughput, during optical modulation the RF output is left open. The reflection from the open end creates a standing wave along the microstripline that enhances the total voltage gain of the modulator.

A microprism is used to evanescently couple optical power into and out of the microdisk. The TE-polarized ( $E$-field parallel to the $z$-axis) optical input power is focused on the microprism through a matched-pair lens system, and the output is collected using a cleaved single-mode fiber. 
Fig. 3(b) shows a photograph of the modulator arrangement showing the microdisk, the microprism, cleaved fiber, metalring RF resonator, and the microstripline. Fig. 3(c) shows the typical optical transmission spectrum of the $\mathrm{LiNbO}_{3}$ microdisk shown in Fig. 3(b). As may be seen, one mode is critically coupled, resulting in zero transmission at resonance. The optical critical coupling is achieved by tuning the incident angle and the waist size of the optical input beam.

The optical transfer function of a single prism-coupled microdisk modulator can be calculated using a general relation for coupling between an optical microresonator and a dielectric waveguide [13]. The transmitted optical power ratio $(T=$ $\left.P_{\text {ot }} / P_{o, \text { in }}\right)$ is written as

$$
T=\frac{a^{2}+|t|^{2}-2 a|t| \cos (\pi k D)}{1+a^{2}|t|^{2}-2 a|t| \cos (\pi k D)}
$$

where $D$ is the microdisk diameter, $k$ is the wave vector of the WG optical resonance, $a=\exp (-\alpha \pi D)$ is the inner circulation factor ( $\alpha$ is the distributed internal loss factor), and $t$ is the coupler's transmission coefficient. The optical coupling factor $\left(\kappa_{O}\right)$ may be expressed as $\left(1-t t^{*}\right)^{1 / 2}$. It is important to notice that all of these parameters except $D$ vary for different WG resonances. Each resonant dip has a Lorentzian shape with full-width half-maximum (FWHM) wavelength $\Delta \lambda_{\mathrm{FWHM}}$ around its resonant wavelength $\lambda_{\text {res }}$ and a loaded optical $Q=$ $\lambda_{\text {res }} / \Delta \lambda_{\mathrm{FWHM}}$ limited by $\kappa_{o}$ and $\alpha$. Loaded $Q$ is estimated as [14]

$$
Q=\frac{\pi^{2} n_{\mathrm{eo}} D \sqrt{t a}}{(1-t a) \lambda_{\mathrm{res}}}
$$

where $n_{\mathrm{eo}}=2.14$ is the unperturbed extraordinary refractive index of $\mathrm{LiNbO}_{3}$. The resonant frequencies associated with each set of optical modes are equally spaced from each other by the optical free spectral range of the microdisk resonator $\left(\Delta \nu_{\mathrm{FSR}}=c / \pi n_{\mathrm{eO}} D\right)$. Critical coupling is equivalent to $N_{0}=0$ (at $\lambda_{\text {laser }}=\lambda_{\text {res }}$ ) and is an ideal condition for self-homodyne photonic mixing because the dc optical noise is limited by the dc component generated in the mixing process, $\left(N_{2} V_{0}^{2} / 4\right)\left(1+m^{2} / 2\right)$.

\section{B. Linear and Nonlinear Modulation}

A microdisk modulator has a very high sensitivity within a limited bandwidth centered at frequencies equal to integral multiples of the $\Delta \nu_{\mathrm{FSR}}$ [10]. The high sensitivity of the modulator is a result of simultaneous RF and optical resonance. Typically, the modulation bandwidth is limited by the optical $Q$. Using a microdisk modulator with $\Delta \nu_{\mathrm{FSR}}=14.6 \mathrm{GHz}$ (Fig. 3), operating in the linear regime, we have measured a signal-to-noise ratio (SNR) of $12 \mathrm{~dB}$ at $-67-\mathrm{dBm} \mathrm{RF}$ input power (corresponding to a sensitivity of $-80 \mathrm{dBm}$ ). We note that, previously, Ilchenko et al. have reported a $150-\mu \mathrm{m}$-thick microdisk linear modulator with an SNR of $14 \mathrm{~dB}$ when excited by $-56-\mathrm{dBm}$ RF power at 9.15 GHz [15].

We can use (9) to estimate the modulated optical power at any RF input voltage $\left(V_{\mathrm{RF}}\right)$ and optical input wavelength $\left(\lambda_{\text {laser }}\right)$ knowing the relation between $V_{\mathrm{RF}}$ and $k$ as follows:

$$
k\left(V_{\mathrm{RF}}\right)=\frac{2 \pi n_{\mathrm{e}}\left(V_{\mathrm{RF}}\right)}{\lambda_{\text {laser }}}=\frac{2 \pi\left[n_{\mathrm{eo}}+\delta n_{\mathrm{e}}\left(V_{\mathrm{RF}}\right)\right]}{\lambda_{\text {laser }}} .
$$

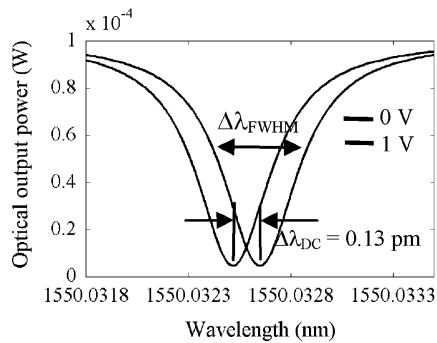

( a )

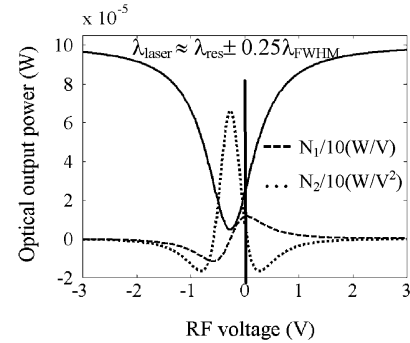

(b)

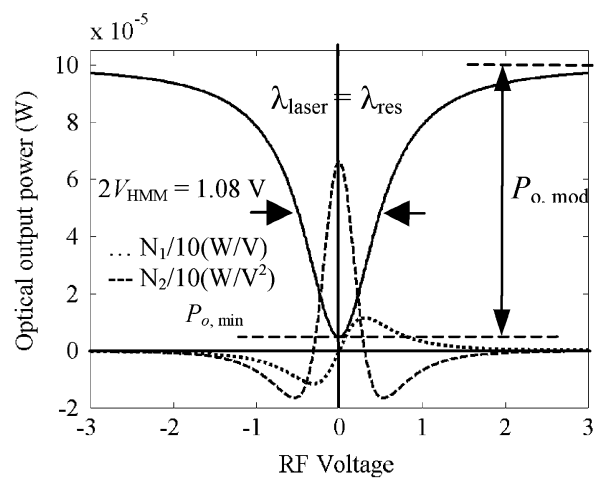

(c)

Fig. 4. (a) Calculated transmitted optical power in the vicinity of a resonant mode at 0 and $1 \mathrm{~V}$ applied de voltage for a 0.4-mm-thick disk with $\beta_{\mathrm{EO}}=$ $0.5, \kappa_{o}=0.095$, and $\alpha=0.0075 \mathrm{~cm}^{-1}$. The optical input power is $1 \mathrm{~mW}$, the insertion loss is $10 \mathrm{~dB}$, and $\lambda_{\text {laser }} \sim 1550 \mathrm{~nm}$. (b) Calculated optical output intensity of the microdisk modulator as a function of RF input voltage $\left(G_{\mathrm{v}}=\right.$ $6)$. The dashed and dotted lines are generated as the first $\left(N_{1}\right)$ and second $\left(N_{2}\right)$ derivatives of the optical transfer function (solid line). The laser wavelength is biased to the linear operation regime where at $V_{\mathrm{RF}}=0, N_{1}$ is maximum and $N_{2}=0$. (c) Calculated optical output intensity of the microdisk modulator biased at the extreme nonlinear operation regime $\left(\lambda_{\text {res }}=\lambda_{\text {laser }}\right)$ versus RF voltage.

Here, $\delta n_{\mathrm{e}}\left(V_{\mathrm{RF}}\right)$ is the electrooptically induced refractive index change given by

$$
\delta n_{e}\left(V_{\mathrm{RF}}\right)=\frac{1}{2} n_{\mathrm{eo}}^{3} r_{33} \frac{\beta_{\mathrm{EO}} \beta_{\mathrm{S}} G_{\mathrm{V}}}{h} \times V_{\mathrm{RF}}
$$

where $\beta_{\mathrm{S}} \sim 0.5$ is a correction factor that accounts for the sinusoidal spatial $E$-field distribution around the ring when its fundamental mode is excited, $\beta_{\mathrm{EO}}$ is an optical-mode-electric-field overlap correction factor, and $\mathbf{r}_{33}=30.8 \times 10^{-12} \mathrm{~m} / \mathrm{V}$ is the linear electrooptic coefficient of $\mathrm{LiNbO}_{3}$ along the $c$-axis. By measuring the resonant wavelength shift $\Delta \lambda_{\mathrm{DC}}$ (called the dc shift) when $1 \mathrm{~V}$ is directly applied to the metal ring, one may estimate $\beta_{\mathrm{EO}}$.

In Fig. 4, the transmitted optical power $\left(P_{\text {ot }}\right)$ for the modulator shown in Fig. 3 is simulated as a function of wavelength and input RF voltage amplitude.

In our simulation, the modulator parameters are chosen to be representative of the experimental values with $Q=3.5 \times 10^{6}$ (corresponding to $\alpha=0.0075 \mathrm{~cm}^{-1}$ and $\kappa_{o}=0.095$ ), $h=$ $400 \mu \mathrm{m}$, and $G_{\mathrm{v}}=6$. The optical input power is $1 \mathrm{~mW}$, the insertion loss is $10 \mathrm{~dB}$, and $\lambda_{\text {laser }} \sim 1550 \mathrm{~nm}$.

Fig. 4(a) shows $P_{\text {ot }}$ as a function of wavelength at 0 - and $1-\mathrm{V}$ dc applied to the ring resonator. For this modulator, the measured value of $\Delta \lambda_{\mathrm{DC}}=0.13 \mathrm{pm} / \mathrm{V}$ corresponds to $\beta_{\mathrm{EO}}=$ 0.53. Fig. 4(b) shows $P_{\text {ot }}$ calculated as a function of input RF voltage $\left(V_{\mathrm{RF}}\right)$. The laser wavelength $\left(\lambda_{\text {laser }}\right)$ is tuned to $\lambda_{\text {res }}+$ 


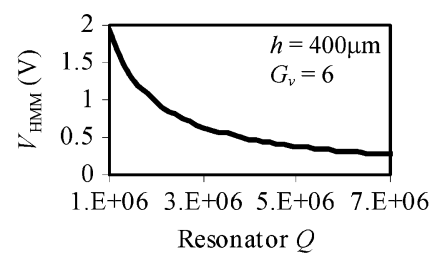

(a)

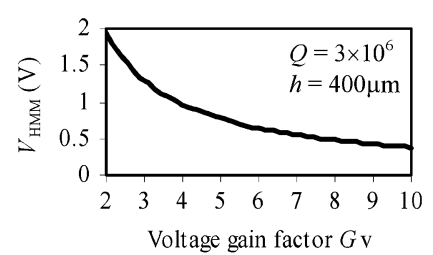

(b)

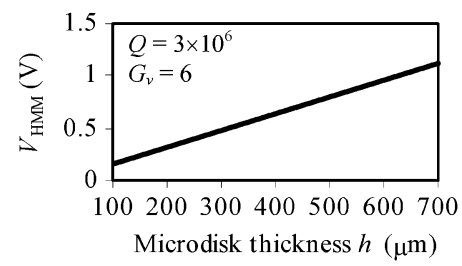

(c)

Fig. 5. Calculated value of $V_{\mathrm{HMM}}$ as a function of: (a) resonator $Q$, (b) voltage gain factor $G_{\mathrm{v}}$, and (c) microdisk thickness $h$ using the electrooptic transfer function.

$\left(\Delta \lambda_{\mathrm{FWHM}} / 4\right)$, where $N_{1}$ is maximized at $V_{\mathrm{RF}}=0$. This is the condition for optimized linear optical intensity modulation using a microdisk modulator. The dashed and dotted lines represent the calculated first $\left(N_{1}\right)$ and second $\left(N_{2}\right)$ derivatives of the microdisk optical transfer function (solid line).

The essential idea in this study is to use nonlinear optical modulation as the key element in a photonic self-homodyne RF receiver. In Fig. 4(c), the laser wavelength is tuned to an optical resonance of the microdisk $\left(\lambda_{\text {res }}=\lambda_{\text {laser }}\right)$ so that, in the absence of an external voltage $\left(V_{\mathrm{RF}}=0\right)$, the transmitted optical power is minimized. At this bias point, $N_{1}$ is zero, and $N_{2}$ is maximized so that the modulator is operating in the extreme nonlinear regime. The sensitivity of the modulator can be quantified by the RF voltage amplitude $V_{\mathrm{HMM}}$ that modulates half of the optical mode power $\left(P_{o, \text { mod }}=P_{o, \text { in }}-P_{o, \text { min }}\right)$. $V_{\mathrm{HMM}}$ is determined by the optical $Q, h, \mathbf{r}_{33}$, and $G_{\mathrm{v}}$.

Fig. 5 shows the calculated values of $V_{\mathrm{HMM}}$ for an $\mathrm{LiNbO}_{3}$ microdisk modulator $\left(\mathbf{r}_{33}=30.8 \times 10^{-12} \mathrm{~m} / \mathrm{V}\right)$ against (a) resonator $Q$, (b) voltage gain factor $G_{\mathrm{v}}$, and (c) microdisk thickness $h$ using the electrooptic transfer function (9).

If $V_{\mathrm{RF}}<0.25 V_{\mathrm{HMM}}$ the microdisk modulator is effectively operating as a square-law optical intensity modulator $\left(\left(N_{2} / 2\right) V_{\mathrm{RF}}^{2}>\left(N_{i} / i !\right) V_{\mathrm{RF}}^{i}, i>2\right)$, as explained in Section II. In this voltage range, the modulation depth is about $12.5 \%$ $(-9 \mathrm{~dB})$. We call this regime the square-law operating regime. $N_{2}$ (which is directly proportional to $P_{o, \text { in }}$ and inversely to $V_{\text {HMM }}$ ) can be calculated using (9), (11), and (12). Fig. 4(c) shows that, for an ideal optical resonance, $V_{\mathrm{HMM}}=0.54 \mathrm{~V}$ and $P_{o, \text { in }}=1 \mathrm{~mW}$ result in $N_{2}=0.67 \mathrm{~mW} / \mathrm{V}^{2}$ for this specific modulator.

\section{COMPARISON BETWEEN MZ AND MICRODISK MOdulator as SQuare-LaW MiXers}

An MZ modulator, biased at minimum transmission, can perform square-law RF mixing in the optical domain similar to a microdisk modulator. Fig. 6(a) and (b) plots the calculated optical output power against the applied RF voltage for an MZ and a microdisk modulator, respectively (solid lines). The dashed

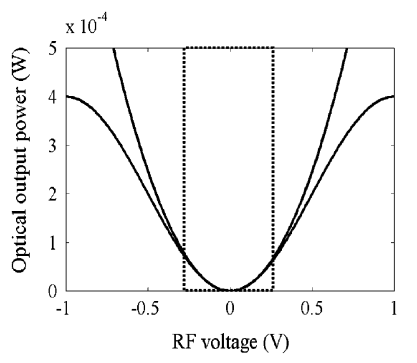

(a)

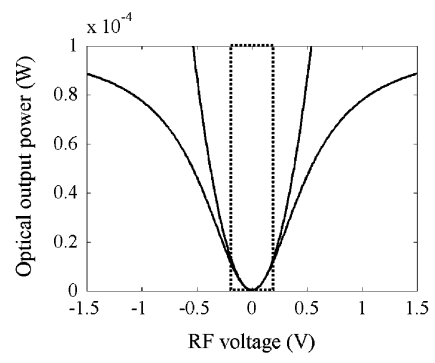

(b)

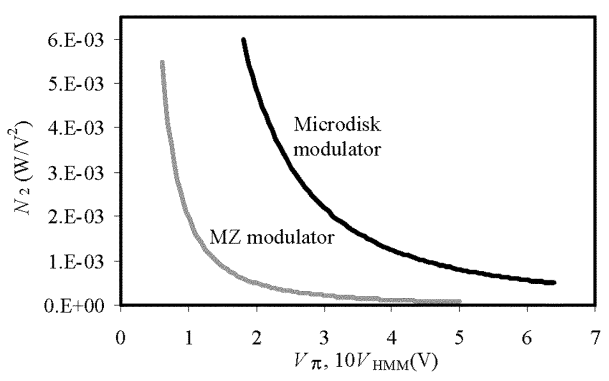

(c)

Fig. 6. (a) Calculated optical output power against the RF voltage for an $\mathrm{MZ}$ modulator with a $V_{\pi}$ of $1 \mathrm{~V}$ and insertion loss of $4 \mathrm{~dB}$. The gray line is the approximated parabola $\left(N_{2} / 2\right) V^{2}$. The dotted blocks show the square-law regime. (b) Calculated optical output power against RF voltage. The microdisk has a $V_{\mathrm{HMM}}$ of $0.55 \mathrm{~V}$ and insertion loss of $10 \mathrm{~dB}$. The optical input power is $1 \mathrm{~mW}$. (c) Calculated value of $N_{2}$ versus $V_{\mathrm{HMM}}$ and $V_{\pi}$ assuming the MZ has an insertion loss of $4 \mathrm{~dB}$ and microdisk modulator has an insertion loss of $10 \mathrm{~dB}$.

curves are the parabolas defined by $\left(N_{2} / 2\right) V^{2}$. The MZ modulator has $V_{\pi}$ of $1 \mathrm{~V}$ and optical insertion loss of $4 \mathrm{~dB}$, and the microdisk modulator has $V_{\mathrm{HMM}}$ of $0.5 \mathrm{~V}$ and optical insertion loss of $10 \mathrm{~dB}$. These numbers have been chosen based on the best reported performance for an $\mathrm{LiNbO}_{3} \mathrm{MZ}$ modulator [16], and the typical response of our $14.6-\mathrm{GHz} \mathrm{LiNbO}_{3}$ microdisk modulator.

In the square-law regime (defined by the dotted boxes), where $V_{\mathrm{RF}}<0.25 V_{\mathrm{HMM}}\left(<0.25 V_{\pi}\right.$ for MZ modulator $)$, the parabolas almost perfectly match the actual response, hence, the strength of the second-order nonlinear modulation can be estimated simply by calculating $N_{2}$. One can use the magnitude of $N_{2}$ to compare the performance of MZ and microdisk modulators as square-law mixers. The received RF signal for wireless communications is below $-30 \mathrm{dBm}$, corresponding to a voltage amplitude of less than $0.01 \mathrm{~V}$. Since the typical value of $V_{\mathrm{HMM}}$ is between $0.2-0.6 \mathrm{~V}$ and a typical value of $V_{\pi}$ is between 1-5 V, our comparison is valid for most wireless applications. Fig. 6(c) shows the calculated value of $N_{2}$ versus $V_{\mathrm{HMM}}$ and $V_{\pi}$ (notice that $V_{\mathrm{HMM}}$ on the $x$-axis is scaled by a factor of ten). Note that the state-of-the-art $\mathrm{LiNbO}_{3} \mathrm{MZ}$ modulator with a $V_{\pi}$ of $1 \mathrm{~V} \mathrm{[16]} \mathrm{has} \mathrm{the} \mathrm{same} \mathrm{nonlinear} \mathrm{modulation} \mathrm{efficiency} \mathrm{as} \mathrm{an}$ $\mathrm{LiNbO}_{3}$ microdisk modulator with a $V_{\mathrm{HMM}}$ of about $0.4 \mathrm{~V}$ that can easily be made using a $300-\mu$ m-thick $\mathrm{LiNbO}_{3}$ microdisk.

More importantly, the insertion loss of the microdisk modulator can be improved without affecting its sensitivity. By contrast, enhanced sensitivity in an MZ modulator is generally accompanied by extra loss associated with longer optical and RF waveguide length. In fact, the 4-dB optical insertion loss for an $\mathrm{MZ}$ modulator with a $V_{\pi}$ of $1 \mathrm{~V}$ is a very optimistic assumption, 


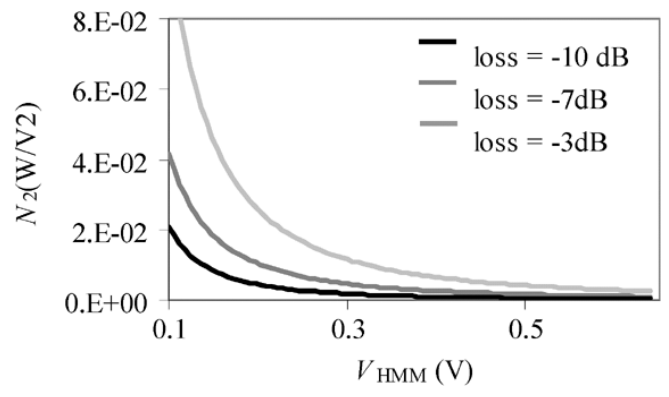

Fig. 7. Simulated magnitude of $N_{2}$ as a function of $V_{\mathrm{HMM}}$ for different values of insertion loss. The optical input power is $1 \mathrm{~mW}$.

while, on the other hand, the 10-dB insertion loss and $V_{\mathrm{HMM}}$ of $0.5 \mathrm{~V}$ for an $\mathrm{LiNbO}_{3}$ microdisk modulator is easily achievable.

Although in most of our experiments the optical insertion loss was around $10 \mathrm{~dB}$, insertion loss as low as $6 \mathrm{~dB}$ has been reported by others [17]. Fig. 7 shows $N_{2}$ versus $V_{\mathrm{HMM}}$ for the $\mathrm{LiNbO}_{3}$ microdisk modulator in Fig. 6(c) for three different values of insertion losses. A typical commercial $10-\mathrm{GHz} \mathrm{MZ}$ modulator has a $V_{\pi}$ of $4.5 \mathrm{~V}$ and an optical insertion loss of about $4 \mathrm{~dB}$, resulting in a $N_{2}$ of $9 \times 10^{-5} \mathrm{~W} / \mathrm{V}^{2}$ at $1-\mathrm{mW}$ optical input power. Our 14.6-GHz $\mathrm{LiNbO}_{3}$ microdisk modulator has a $V_{\mathrm{HMM}}$ of $0.6 \mathrm{~V}$ and insertion loss of $10 \mathrm{~dB}$. This results in a $N_{2}$ of about $7 \times 10^{-4} \mathrm{~W} / \mathrm{V}^{2}$ (at $1-\mathrm{mW}$ optical input power) that is eight times larger than that of the MZ modulator.

The modulation bandwidth limitation imposed by the optical FSR and quality factor is the main disadvantage of the microdisk modulator compared to the broad-band response of the MZ modulator. For example, the modulator microdisk modulator shown in Fig. 3 can modulate light within a $150-\mathrm{MHz}$ bandwidth centered around $14.6 \mathrm{GHz}, 2 \times 14.6 \mathrm{GHz}$, and so on.

\section{DOWn-CONVERSION In A MicrodisK MOdUlator}

\section{A. Single-Tone Down-Conversion}

In our initial experiments, we use a single-tone baseband signal to study the effect of RF modulation index $(m)$ and RF power on down-conversion efficiency and its linearity. Fig. 8 is a schematic diagram of the experimental arrangement. The modulator uses a $400-\mu$ m-thick $\mathrm{LiNbO}_{3}$ microdisk of 3-mm diameter and an FSR of $\Delta \nu_{\mathrm{FSR}}=14.6 \mathrm{GHz}$.

The optical source is a tunable single-mode laser with $0.05-\mathrm{pm}$ wavelength resolution and a linewidth of less than $0.5 \mathrm{MHz}$. The laser wavelength is always tuned to the minimum of the selected transmission dip to maximize the second-order nonlinear modulation strength $\left(N_{2}\right)$. The RF signal is a $10-\mathrm{MHz}$ single-tone baseband signal mixed with a 14.6-GHz RF carrier in a double-balanced RF mixer. By dc-biasing the IF port of the mixer, we can control the modulation index $(m)$ and magnitude of the transmitted power at the carrier frequency. The RF signal is fed to the microdisk modulator through a bandpass RF filter with $1-\mathrm{GHz}$ bandwidth around $14.5 \mathrm{GHz}$ to ensure that any nonlinear products generated in the RF components are filtered out. The optical output is detected in an amplified photodetector with a bandwidth of $150 \mathrm{MHz}$ and responsivity of $3 \mathrm{mV} / \mu \mathrm{W}$ $(R=0.8 \mathrm{~A} / \mathrm{W}$ and transimpedance $=3700 \Omega)$.

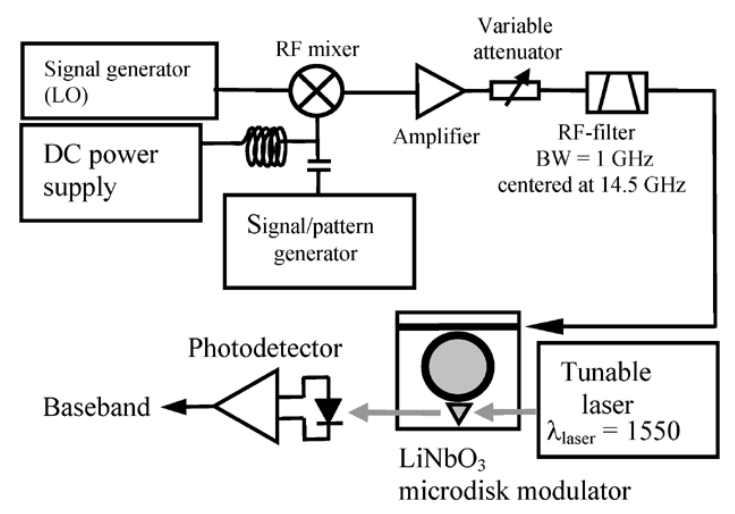

Fig. 8. Schematic diagram of the experimental arrangement used for photonic $\mathrm{RF}$ down-conversion measurements. The RF modulation index $(m)$ is tuned using the dc bias on the mixer. The laser is a tunable single-mode laser with a resolution of $0.1 \mathrm{pm}$ and linewidth of less than $0.5 \mathrm{MHz}$. The RF filter eliminates any low-frequency component generated due to nonlinearities in RF devices. The LO frequency is $14.6 \mathrm{GHz}$, which is equal to the optical FSR of the microdisk modulator.

Fig. 9(a) shows the measured down-converted optical power at $10 \mathrm{MHz}$ as a function of the modulation index $m$ and for three resonances with different quality factors. The modulation index is tuned to the desired values by changing the dc bias applied to the mixer. The total received RF power is about $-15 \mathrm{dBm}$ that, corresponding to $V_{0}=0.05 \mathrm{~V}$ [see (3)]. The $V_{\mathrm{HMM}}$ is around $0.8 \mathrm{~V}$ for the optical resonance used, so $V_{0}<0.25 V_{\mathrm{HMM}}$ guarantees device operation in the square-law regime.

As may be seen in Fig. 9(a), down-conversion efficiency is maximized around $m=0.8$, which is in very good agreement with the simulated curve for an ideal square law mixer [see Fig. 2(a)]. Also, as anticipated, the amount of down-converted power increases as we increase the optical $Q$ (a larger $Q$ results in a larger $V_{\mathrm{HMM}}$ and therefore a larger $N_{2}$ ). Also, as anticipated, the amount of down-converted power is larger for larger values of optical $Q$. The different values of $Q$ in Fig. 9(a) correspond to different optical resonances that are chosen by tuning the laser wavelength. To make sure that the down-conversion efficiency is only affected by the optical $Q$, we have verified that the chosen modes have the same electrooptical response (i.e., of $\Delta \lambda_{\mathrm{DC}}$ for a given voltage).

Fig. 9(b) plots the down-converted optical power against the total RF input power when $m=0.8$. The black circles are the experimental data while the white circles and dashed line comprise the simulated data. The inset shows the optical resonance selected for nonlinear modulation. The black arrow indicates the location of the laser wavelength $\lambda_{\text {laser }}$. In this case, the optical resonance has $Q=2.5 \times 10^{6}$ and a $N_{2}$ coefficient of $0.23 \mathrm{~mW} / \mathrm{V}^{2}\left(V_{\mathrm{HMM}}=0.7 \mathrm{~V}\right)$. The simulated data in Fig. $9(\mathrm{~b})$ are calculated using $m N_{2} V_{0}^{2} / 2$ and knowing that the total average RF power of a single-tone modulated RF carrier (3) is given by $P_{\mathrm{RF}}=V_{0}^{2}\left(1+m^{2} / 2\right) / 100$ [this can be easily calculated by integrating the average power of the RF signal in (3)]. The good agreement between experimental and calculated data verifies the validity of (8). To evaluate the linearity of the down-conversion process, we have measured the detected power at the second and third harmonic of the baseband signal (20 and $30 \mathrm{MHz}$, respectively). In a perfect square-law modulator, the 

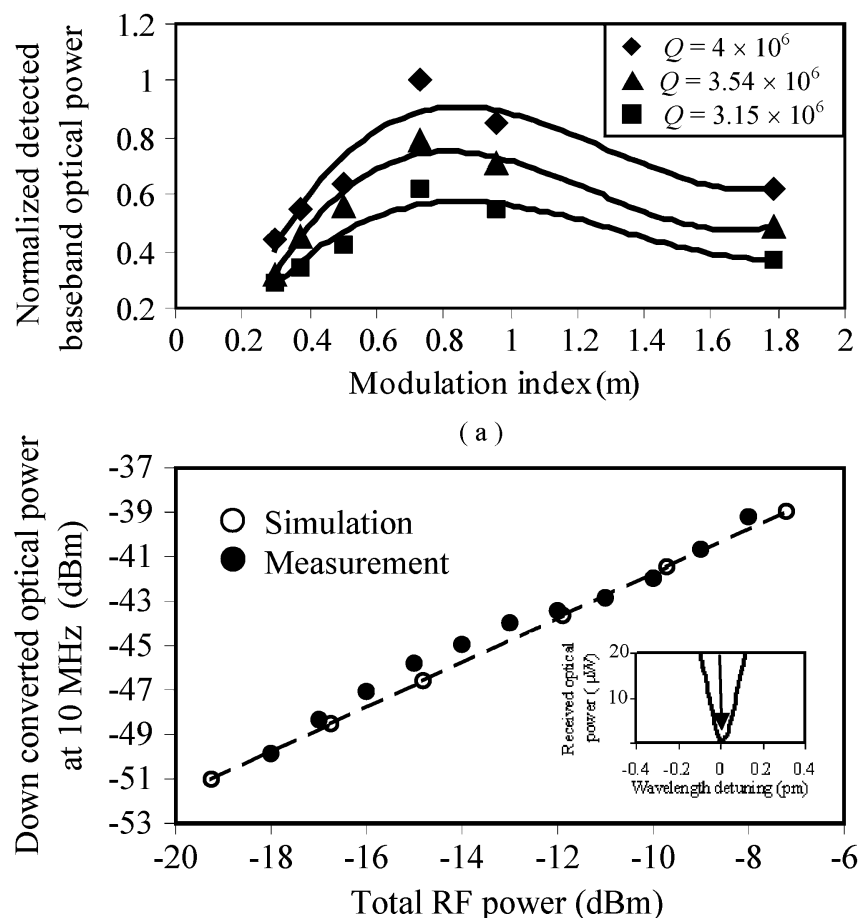

( b )

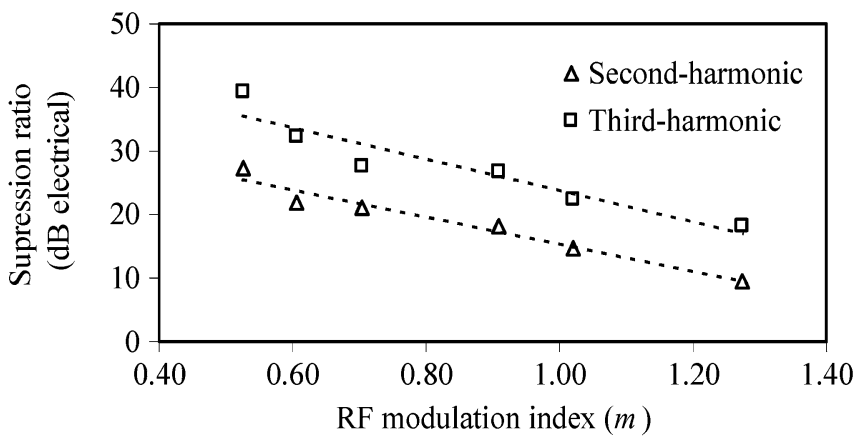

(c)

Fig. 9. (a) Measured baseband modulated (10 MHz) optical output power against $m$ for three optical modes with different optical quality factors. The solid lines are the calculated responses. (b) The measured and calculated baseband modulated optical power versus total RF input power. The inset shows the optical spectrum of the WG resonance chosen for down-conversion $\left(Q=2.7 \times 10^{6}\right)$. (c) Measured second- and third-harmonic suppression ratios (electrical) against $m$. The dotted lines are to guide the eye.

third harmonic should be absent but the chosen optical resonance does not have an ideal symmetric shape and so generates odd harmonics.

Fig. 9(c) shows the harmonic suppression ratio against $m$. As predicted [see Fig. 2(b)], the suppression ratio decreases as $m$ increases. At $m=0.8$, the second-harmonic suppression ratio is approximately $17 \mathrm{~dB}$ (electrical).

\section{B. Down-Conversion of Digital Data}

For experimental demonstration of data transmission, we use the arrangement in Fig. 8 but replace the signal generator with a nonreturn-to-zero (NRZ) pattern generator and the photodetector with a digital photoreceiver.

Fig. 10(a) shows the measured frequency spectrum of the input RF signal and the down-converted signal after detection. The photoreceiver has a $-3-\mathrm{dB}$ frequency bandwidth of
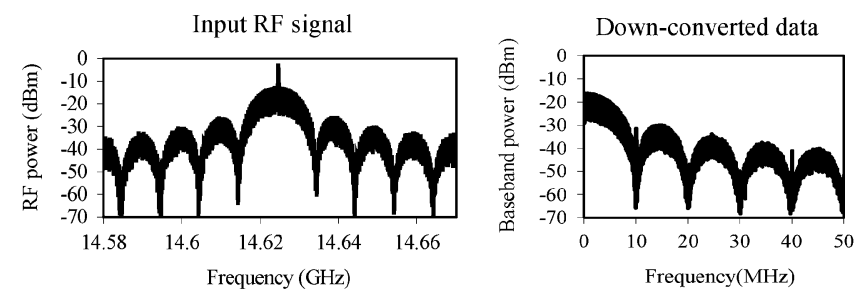

( a )

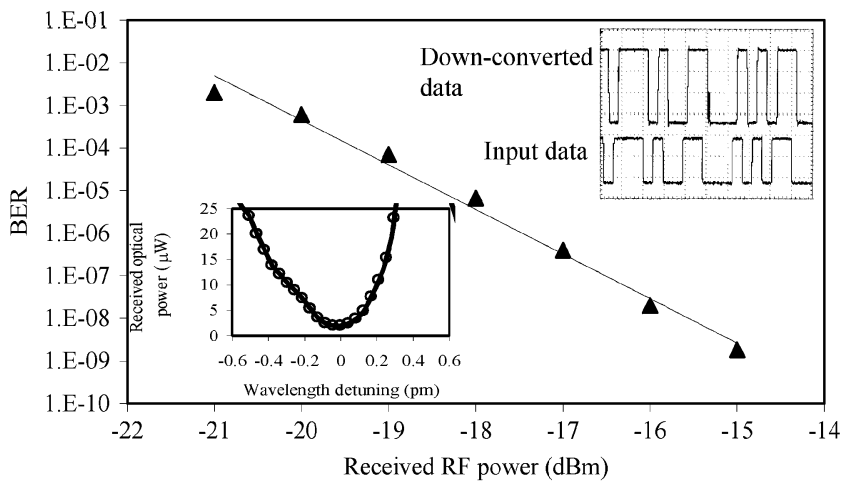

(b)
$10 \mathrm{Mb} / \mathrm{s}$

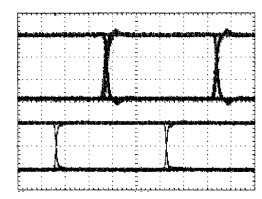

Time, $t(25 \mathrm{~ns} / \mathrm{div})$
$50 \mathrm{Mb} / \mathrm{s}$

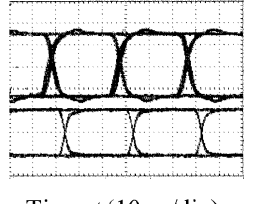

Time, $t$ (10 ns/div)

(c)
Fig. 10. Measurement results of photonic data down-conversion in an $\mathrm{LiNbO}_{3}$ microdisk modulator. (a) The frequency spectrum of the input RF signal and down-converted signal. The RF carrier frequency is $14.6 \mathrm{GHz}$, and it is modulated by a $10-\mathrm{Mb} / \mathrm{s} 2^{7}-1 \mathrm{NRZ}$ PRBS bit stream. (b) BER sensitivity of the photonic RF receiver. The RF power is the measured RF power within $10-\mathrm{MHz}$ bandwidth centered around $14.6 \mathrm{GHz}$. The right inset shows the input and detected data in the time domain. The left inset shows the optical spectrum of the selected WG resonance. (c) Measured input (bottom) and output (top) eye diagrams at 10,50 , and $100 \mathrm{Mb} / \mathrm{s}$. The received $\mathrm{RF}$ power is about $-17 \mathrm{dBm}$.

$120 \mathrm{MHz}$ and a sensitivity of $-34.5 \mathrm{dBm}$. The carrier frequency is $14.62 \mathrm{GHz}$ and the baseband signal is a $10-\mathrm{Mb} / \mathrm{s} \mathrm{NRZ} 2^{7}-1$ pseudorandom bit stream (PRBS).

Fig. 10(b) shows the measured bit error rate (BER) against the total RF input power. The received RF power is defined as the measured RF power within the data bandwidth centered around $14.6 \mathrm{GHz}$. The left inset shows the spectrum of the optical resonance with $Q=2 \times 10^{6}$. The inset on the right shows the input and down-converted data in the time domain.

In Fig. 10(c), the measured input (bottom) and output (top) eye diagrams at 10,50 , and $100 \mathrm{Mb} / \mathrm{s}$ are shown. In this particular case, the maximum data rate is limited by the optical $Q$ to approximately $150 \mathrm{Mb} / \mathrm{s}$.

\section{WIRELESS TRANSMISSION}

In this section, we present the preliminary results of employing the photonic receiver in a short wireless link. In a one-directional indoor wireless link, the transmitter antenna can have a fixed position while the receiver antenna is free to move in the area illuminated by the transmitter. In our 


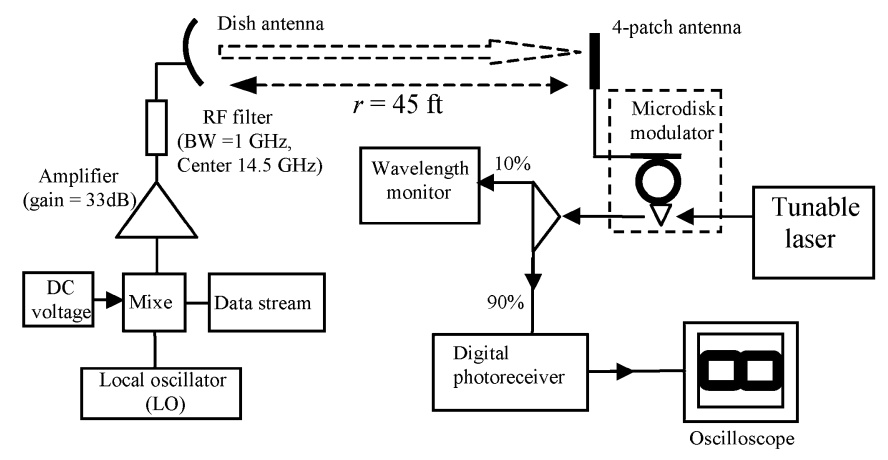

Fig. 11. Schematic diagram of the experimental wireless link. The parabolic antenna is used as the transmitter. The antenna has a diameter of $1 \mathrm{ft}$ and a gain of $33 \mathrm{~dB}$. The four-patch antenna has a gain of $10 \mathrm{~dB}$ and is directly connected to the microdisk modulator through a coaxial cable.

experiment, we use a parabolic transmitter antenna and a small four-patch receiver antenna. The transmit antenna has a diameter of $1 \mathrm{ft}$, gain of $G_{\mathrm{t}}=33 \mathrm{~dB}$, and 3-dB bandwidth of $110 \mathrm{MHz}$ around $14.7 \mathrm{GHz}$. The beam full-width angular spread is about $3.5^{\circ}$. The four-patch antenna has an area of one square inch, gain of $G_{\mathrm{r}}=10 \mathrm{~dB}$, and 3-dB bandwidth of $900 \mathrm{MHz}$ around 14.6 GHz. Fig. 11 shows a schematic diagram of the wireless link. The transmitted carrier signal is amplified and then fed to the parabolic antenna through a bandpass filter. The modulation format and the power of the RF signal are controlled by LO power and the dc bias on the mixer. The four-patch array receiver antenna is located $45 \mathrm{ft}$ from the transmitter and is directly attached to the microdisk modulator with a coaxial cable. The digital photoreceiver in this experiment has a sensitivity of $-40 \mathrm{dBm}$ (for a BER of $10^{-9}$ ) and digital bandwidth of $52 \mathrm{Mb} / \mathrm{s}$.

In our experiment, the input RF power to the transmitter antenna is $19 \mathrm{dBm}$ and the received power is $-20 \mathrm{dBm}$. The measured value of the received RF power is in good agreement with the Friis formula $P_{\mathrm{r}}=P_{\mathrm{t}} G_{\mathrm{r}} G_{\mathrm{t}} \lambda^{2} /(4 \pi r)^{2}$, where $P_{\mathrm{r}}$ is the received power, $P_{\mathrm{t}}$ is the input power to the transmit antenna, $\lambda$ is the RF wavelength $(2.1 \mathrm{~cm})$, and $r$ is the distance between two antennae $(\sim 14 \mathrm{~m})$.

Fig. 12(a) shows the spectrum of the optical resonance chosen for photonic down-conversion. The optical input power is $1 \mathrm{~mW}$ while the maximum transmitted optical power is about $30 \mu \mathrm{W}$, corresponding to an optical coupling efficiency of $3 \%$.

Fig. 12(b) shows the electrooptic transfer function of the optical resonance with $V_{\mathrm{HMM}}=0.6 \mathrm{~V}$. The solid line is the estimated parabolic behavior in the square-law regime. Fig. 12(c) shows the measured input (bottom) and output (top) eye diagrams after photonic down-conversion from the $14.6-\mathrm{GHz}$ carrier. The data is NRZ $2^{7}-1$ PRBS and the measured BER is $10^{-6}$ at $-20-\mathrm{dBm}$ received $\mathrm{RF}$ power for both 10 - and $50-\mathrm{Mb} / \mathrm{s}$ data streams. Knowing the RF modulation index, we can estimate the baseband modulated optical power using (6). At $-20-\mathrm{dBm}\left(V_{0}=0.032 \mathrm{~V}\right)$ received RF power, the baseband modulated optical power is about $-32 \mathrm{dBm}$. Although the sensitivity of the digital photoreceiver is $-40 \mathrm{dBm}$, the baseband optical noise generated by laser RIN, microdisk thermal noise, and detector shot noise results in a BER of $10^{-6}$.

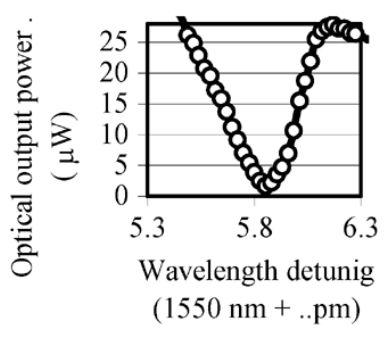

(a)

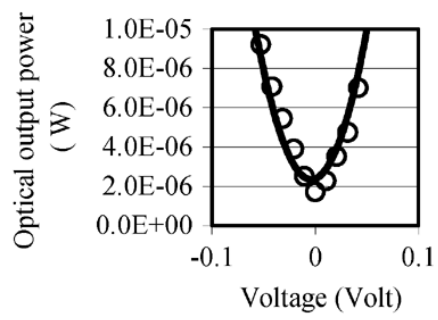

(b)
$10 \mathrm{Mb} / \mathrm{s}$

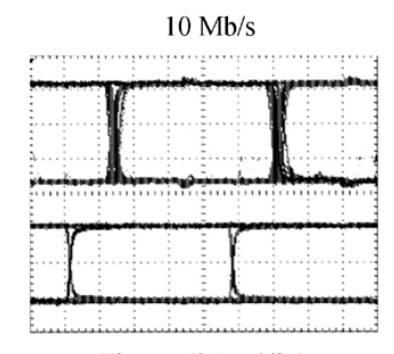

Time, $t(25 \mathrm{~ns} / \mathrm{div})$

( c $)$
Fig. 12. (a) Spectrum of the optical resonance chosen for photonic down-conversion. (b) Electrooptical response of the optical output power. The solid line is the estimated parabolic behavior at square-law regime. (c) Measured input (bottom) and output (top) eye diagrams after photonic down-conversion from a 14.6-GHz carrier. The data is NRZ $2^{7}-1$ PRBS and the measured BER is $10^{-6}$ at $-20-\mathrm{dBm}$ received RF power for both 10 - and $50-\mathrm{Mb} / \mathrm{s}$ data.

\section{MicRodisk Photonic ReCEIVER SENSITIVITY}

An accurate estimate of the sensitivity of the photonic RF receiver requires a detailed analysis of the optical and electrical noise sources in the system, which is beyond the scope of this paper. Nevertheless, we can identify the critical parameters that determine the sensitivity by a simple analysis. The insight we obtain is helpful for optimizing the receiver performance because it identifies the critical parameters.

The gain in the photonic receiver is mainly provided by the RF-to-optical conversion in the modulator and optical-to-electrical baseband conversion in the photoreceiver. Therefore, the total gain is essentially a combination of the modulator and photoreceiver sensitivity. For every photonic down-conversion method, we can define the RF-to-baseband optical gain as

$$
G_{\mathrm{RF}, \mathrm{OB}}=\frac{\text { Baseband modulated optical power }}{\text { Received RF power }} \text {. }
$$

The baseband modulated optical power is directly proportional to $N_{2}$, which is determined by the maximum optical output power and $V_{\mathrm{HMM}}$. Since maximum output power is equal to optical insertion loss times $P_{o}$,in, optical insertion loss, optical input power, and $V_{\mathrm{HMM}}$ are the determining factors for the down-conversion efficiency of the microdisk photonic mixer. Thus, the overall sensitivity of the self-homodyne microdisk receiver can be viewed as a function of all of the above-mentioned parameters plus the sensitivity of the digital photoreceiver.

Sensitivity of a receiver is defined as the minimum detectable RF power at the receiver input such that there is a sufficient SNR 


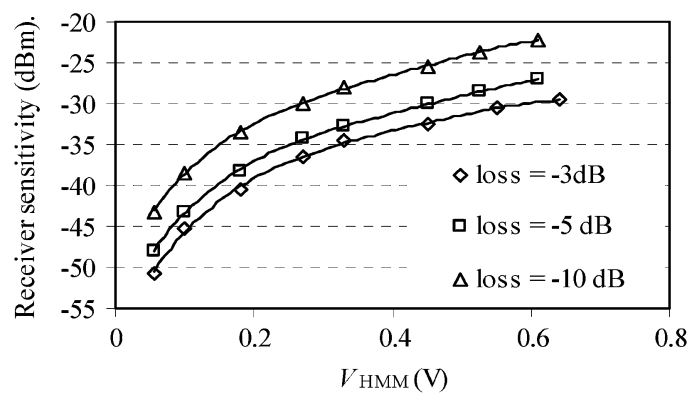

( a )

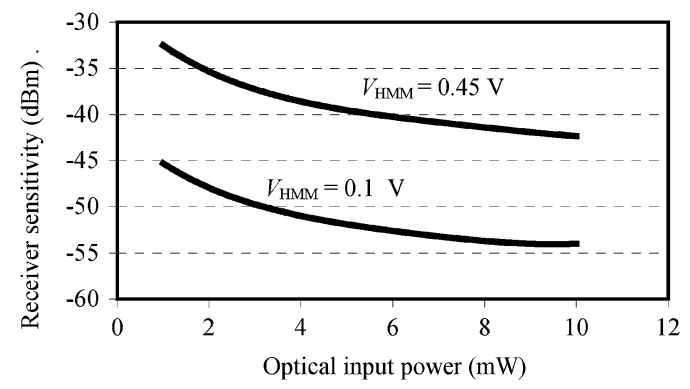

(b)

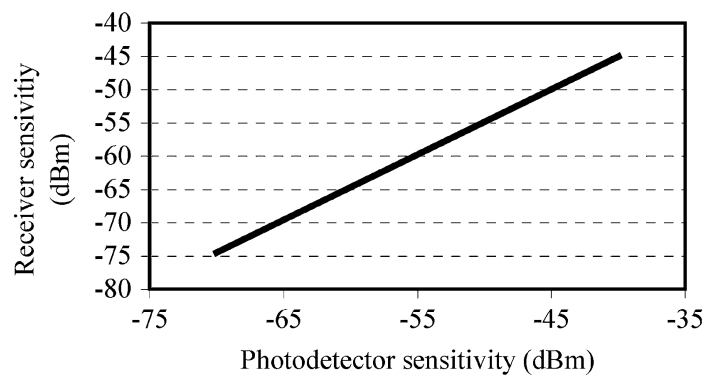

(c)

Fig. 13. (a) Calculated receiver sensitivity against $V_{\text {HMM }}$ for three different values of optical insertion loss $(-3,-5$, and $-10 \mathrm{~dB})$. The optical input power $\left(P_{o, \text { in }}\right)$ is $1 \mathrm{~mW}$, and the sensitivity of the photoreceiver is $-40 \mathrm{dBm}$. (b) Calculated receiver sensitivity against optical input power $\left(P_{o, \text { in }}\right)$ for two microdisk modulators with $V_{\mathrm{HMM}}$ of 0.45 and $0.1 \mathrm{~V}$, respectively. The insertion loss is $-3 \mathrm{~dB}$. Again, the sensitivity of the digital photoreceiver is $-40 \mathrm{dBm}$. (c) Calculated receiver sensitivity against sensitivity of the digital photoreceiver for an optical input power $\left(P_{o, \text { in }}\right)$ of $1 \mathrm{~mW}$, insertion loss of $-3 \mathrm{~dB}$, and $V_{\mathrm{HMM}}$ of $0.1 \mathrm{~V}$.

at the output of the receiver for a given application. Each wireless standard may therefore require a different sensitivity. Current wireless links are mainly digital, so the minimum SNR is translated to the maximum BER that is required in the link. In a digital photonic receiver, it is convenient to use a digital photoreceiver to detect the data-modulated optical power because it contains all of the digital electronic circuitry integrated with the photodiode. The sensitivity $\left(S_{\mathrm{d}}\right)$ of a digital photoreceiver is defined as the minimum data-modulated optical power required to obtain a particular BER $\left(10^{-9}\right.$ for the receiver used in this study) in the output.

Assuming that the maximum BER in the wireless link is the same as the BER at which $S_{\mathrm{d}}$ is measured, the sensitivity of the photonic wireless receiver can be written as

$$
\text { Receiver sensitivity }=\frac{S_{\mathrm{d}}}{G_{\mathrm{RF}, \mathrm{OB}}} .
$$

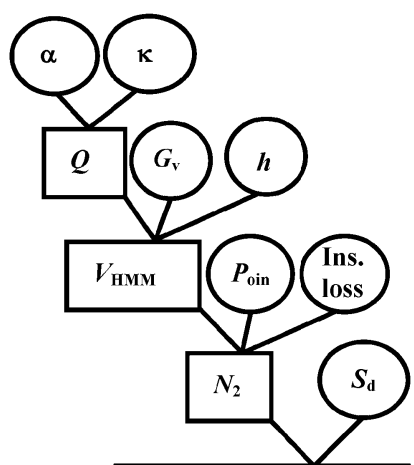

Receiver sensitivity $\boldsymbol{\alpha}$ : Intrinsic loss

$\boldsymbol{\kappa}$. Optical coupling coefficient

$\boldsymbol{Q}$ : Loaded optical quality factor

$\boldsymbol{G}_{\mathbf{v}}:$ RF resonator voltage gain

$\boldsymbol{h}$ : Microdisk thickness

$V_{\text {нмм: Half max modulation RF voltage }}$ amplitude

$\boldsymbol{P}_{\text {oin }}$ : Optical input power

$\mathrm{N}_{2}$ : Second derivative of the electro-

optical transfer function at resonance

$S_{\mathrm{d}}$ : Photodetector sensitivity

Receiver sensitivity: Minimum

received RF power for a certain BER
Ins. loss: Optical insertion loss

Fig. 14. Hierarchy and relation among parameters that determine the overall sensitivity of the microdisk receiver. Circles represent independent parameters and rectangles are functions of the independent parameters.

We can estimate the overall sensitivity of the photonic receiver using (14). In this case, the total RF input power that results in a baseband modulated optical power equal to the sensitivity of the digital photoreceiver is the minimum received power or sensitivity of the wireless receiver (the sensitivity of the digital photoreceiver and the wireless RF receiver are defined based on the same BER).

In this example, we assume a single-frequency baseband and an optimized RF modulation index of $m=0.8$. Fig. 13(a) shows the calculated receiver sensitivity against $V_{\mathrm{HMM}}$ for three different values of optical insertion loss. The optical input power $\left(P_{o, \text { in }}\right)$ is $1 \mathrm{~mW}$, and the sensitivity of the photoreceiver is $-40 \mathrm{dBm}$. Fig. 13(b) shows the calculated receiver sensitivity against optical input power $\left(P_{o, \text { in }}\right)$ for two microdisk modulators with $V_{\mathrm{HMM}}$ of 0.45 and $0.1 \mathrm{~V}$. Again, the sensitivity of the digital photoreceiver is $-40 \mathrm{dBm}$.

Fig. 13(c) shows the calculated receiver sensitivity against the sensitivity of the digital photoreceiver. The optical input power $\left(P_{o, \text { in }}\right)$ is $1 \mathrm{~mW}$ and $V_{\mathrm{HMM}}$ is $0.1 \mathrm{~V}$. As may be seen, a combination of low insertion loss $(<3 \mathrm{~dB})$, sensitive digital photoreceiver $(<-65 \mathrm{~dB})$, and efficient photonic mixing $\left(V_{\mathrm{HMM}}<\right.$ $0.2 \mathrm{~V})$ results in a dramatic improvement in wireless receiver sensitivity $(<-70 \mathrm{dBm})$.

Fig. 14 summarizes the hierarchy and the relation among all of the parameters in the system and the overall sensitivity of the photonic receiver. The parameters in circles are independent and can be improved within the limits of the available technology. Rectangles indicate secondary parameters that are functions of the independent parameters.

We can also increase the sensitivity by employing a bandpass optical filter after the microdisk modulator. A filter with a bandwidth of less than $4 f_{\mathrm{RF}}$ can eliminate high-frequency optical components around $2 f_{\mathrm{RF}}$ and therefore reduce the noise generated by the optical power at these frequencies in the slow-speed photoreceiver.

\section{CONCLUSION}

We have shown that there is an opportunity for electrooptic microresonators to exploit the electrooptical nonlinearity for 
RF signal processing. A self-homodyne photonic RF receiver based on an $\mathrm{LiNbO}_{3}$ microdisk modulator has been described. Down-conversion occurs in the optical domain through nonlinear modulation, thereby eliminating the need for a RF LO and mixer. Experimental verification of the receiver architecture has been achieved by measuring BER and eye diagrams using signals down-converted from a data-modulated transmitted carrier $\mathrm{RF}$ signal with $f_{\mathrm{RF}}=14.6 \mathrm{GHz}$. The performance of the photonic receiver has been successfully tested in a short-distance wireless link by transmitting up to $50-\mathrm{Mb} / \mathrm{s}$ data on a $14.6-\mathrm{GHz}$ carrier.

Although a traveling-wave MZ modulator can also function as a photonic mixer, at high carrier frequencies where high sensitivity dominates the demand for multicarrier frequency operation, the resonant microdisk modulator clearly outperforms the MZ modulator.

The microdisk modulator and the photonic self-homodyne architecture have the potential to be incorporated into a photonic integrated circuit by using alternative electrooptic materials (such as polymers or compound semiconductors). Reducing the disk diameter will extend the carrier frequency into the millimeter-wave regime enabling this receiver architecture to be exploited for future indoor millimeter-wave wireless systems.

Current microdisk technology may not provide the reliability and sensitivity offered by electronic mixing (especially at frequencies below $30 \mathrm{GHz}$ ). As mentioned in Section II, the electronic diode and microdisk photonic mixer can be compared by evaluating $G_{\mathrm{d}}^{\prime}$ for the diode and $R N_{2}$ for the microdisk photonic mixer. At low frequencies (below $5 \mathrm{GHz}$ ), typically $G \prime_{\mathrm{d}} \approx$ $10^{-2} \mathrm{~A} / \mathrm{V}^{2}$ [9], [18], which is 100 times larger than $R N_{2}$ for our $\mathrm{LiNbO}_{3}$ microdisk mixer and a photodetector with $R \approx$ $0.9 \mathrm{~A} / \mathrm{W}$. However, with increasing carrier frequency, the sensitivity of electronic diodes falls and its fabrication cost grows. At frequencies above $10 \mathrm{GHz}$, only special diodes made of compound semiconductor materials (such as modified barrier integrated diodes) may provide the appropriate sensitivity. Also, it has been shown that the traditionally accepted view of the electronic diodes as true square-law detectors at low power levels is correct only under restrictive conditions [18]. By way of contrast, at input voltages smaller than $0.25 V_{\mathrm{HMM}}$, the parabolic transfer function of the microdisk resonator results in a true square-law behavior independent of the external circuitry.

Wavelength stability is a common issue in any system that employs optical resonators. Likewise, operating in the square-law regime in our system relies on the alignment between the laser wavelength $\left(\lambda_{\text {laser }}\right)$ and the microdisk resonant wavelength $\left(\lambda_{\text {res }}\right)$. We have been able to perform most measurements presented in this study with our commercial laser source and at room temperature, but long-term stability of the receiver demands control systems that can lock the laser wavelength to a cavity resonance. Such control systems have been already developed to lock commercial lasers to high- $Q\left(>10^{7}\right)$ resonance of microtoroidal resonators [19]. We believe that, given the advances in control systems, this issue can be resolved by adding a compact electronic control circuit to the microdisk modulator/receiver.

Assuming continued development of photonic materials and fabrication techniques, in the future, the proposed architecture may be able to compete with its electronic counterpart through reduced cost, reduced complexity, and better sensitivity in the millimeter-wave regime. We have identified the critical parameters of the resonant photonic receiver that may be used to establish a base for further research in this area.

\section{ACKNOWLEDGMENT}

The authors would like to thank I. Gheorma, for his help with patch antenna design and testing.

\section{REFERENCES}

[1] B. Vidal, V. Polo, J. L. Corral, and J. Marti, "Efficient architecture for WDM photonic microwave filters," IEEE Photon. Technol. Lett., vol. 16, no. 1, pp. 257-259, Jan. 2004.

[2] P. O. Hedekvist, B.-E. Olsson, and A. Wiberg, "Microwave harmonic frequency generation utilizing the properties of an optical phase modulator," J. Lightw. Technol., vol. 22, no. 3, pp. 882-886, Mar. 2004.

[3] A. Hirata, M. Harada, and T. Nagatsuma, "120-GHz wireless link using photonic techniques for generation, modulation, and emission of millimeter-wave signals," J. Lightw. Technol., vol. 21, no. 10, pp. 2145-2153, Oct. 2003.

[4] G. K. Gopalakrishnan, W. K. Burns, and C. H. Bulmer, "Microwaveoptical mixing in $\mathrm{LiNbO}_{3}$ modulators," IEEE Trans. Microw. Theory Tech., vol. 41, no. 12, pp. 2383-2391, Dec. 1993.

[5] Y. Shi, W. Wang, and J. H. Bechtel, "High-isolation photonic microwave mixer/link for wide-band signal processing and transmission," J. Lightw. Technol., vol. 21, no. 5, pp. 1224-1232, May 2003.

[6] M. Tsuchiya and T. Hoshida, "Nonlinear photodetection scheme and its system applications to fiber-optic millimeter-wave wireless down-links," IEEE Trans. Microw. Theory Tech., vol. 47, no. 7, pp. 1342-1350, Jul. 1999.

[7] A. A. Abidi, "Direct-conversion radio transceivers for digital communications," IEEE J. Solid-State Circuits, vol. 30, no. 12, pp. 1399-1410, Dec. 1995.

[8] Y. Shoji, K. Hamaguchi, and H. Ogawa, "Millimeter-wave remote selfheterodyne system for extremely stable and low cost broad-band signal transmission," IEEE Trans. Microw. Theory Tech., vol. 50, no. 6, pp. 1458-1468, Jun. 2002.

[9] D. M. Pozar, Microwave Engineering. New York: Wiley, 1998.

[10] D. A. Cohen, M. Hossein-Zadeh, and A. F. J. Levi, "High- $Q$ microphotonic electro-optic modulator," Solid State Electron., vol. 45, pp. 1577-1589, 2001.

[11] M. Hossein-Zadeh and A. F. J. Levi, "A new electrode design for microdisk optical modulator," CLEO Tech. Dig., pp. 863-865, Jun. 2003.

[12] — , "Mb/s data transmission over a RF fiber optic link using a $\mathrm{LiNbO}_{3}$ microdisk optical modulator," Solid State Electron., vol. 46, pp. 2173-2178, 2002.

[13] A. Yariv, "Universal relations for coupling of optical power between microresonators and dielectric wave guides," Electron. Lett., vol. 41, pp. 321-322, 2000.

[14] J. Verdeyen, Laser Electronics, 2nd ed. Englewood Cliffs, NJ: Prentice-Hall, 1989.

[15] V. S. Ilchenko, A. A. Savchenkov, A. B. Matsko, and L. Maleki, "Submicrowatt photonic microwave receiver," IEEE Photon. Technol. Lett., vol. 14, no. 11, pp. 1602-1604, Nov. 2002.

[16] M. Sugiyama, M. Doi, S. Taniguchi, T. Nakazawa, and H. Onaka, "Lowdrive voltage $\mathrm{LiNbO}_{3} 40 \mathrm{~Gb} / \mathrm{s}$ modulator," IEEE LEOS Newslett., vol. 17, no. 1, pp. 12-13, Feb. 2003.

[17] A. A. Savchenkov, V. S. Ilchenko, A. B. Matsko, and L. Maleki, "Highorder tunable filters based on a chain of coupled crystalline whispering gallery mode resonators,' IEEE Photon. Technol. Lett., vol. 17, no. 1, pp. 136-138, Jan. 2005.

[18] R. G. Harrison and X. Le Polozec, "Nonsquarelaw behavior of diode detectors analyzed by the Ritz-Galerkin method," IEEE Trans. Microw. Theory Tech., vol. 42, no. 5, pp. 840-846, May 1994.

[19] T. Carmon, T. J. Kippenberg, L. Yang, H. Rokhsari, S. M. Spillane, and K. J. Vahala, "Feedback control of ultra-high- $Q$ microcavities: Application to micro-Raman lasers and microparametric oscillators," Opt. Express, vol. 13, pp. 3558-3566, May 2005. 


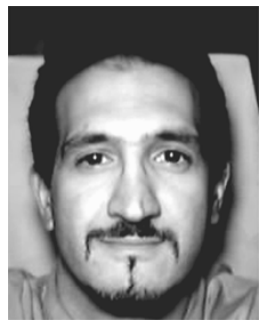

Mani Hossein-Zadeh ( $\left.S^{\prime} 00-M^{\prime} 05\right)$ received the B.S. and M.S. degrees in physics from the Sharif University of Technology, Tehran, Iran, in 1995 and 1997, respectively, and the M.S. and Ph.D. degrees in electrical engineering from the University of Southern California (USC), Los Angeles, in 2001 and 2004, respectively.

From 1995 to 1998, he was a Research Assistant with the Medical Physics Laboratory, Sharif University of Technology, where he was involved with experimental nonlinear optics and laser systems. From 1999 to 2005, he was a Research Assistant with the Advanced Network Technology Laboratory, USC, where he performed research on microwave photonics, especially resonant microdisk modulators and photonic RF receiver design. He is currently a Post-Doctoral Scholar with the Vahala Research Group, California Institute of Technology, Pasadena.

Dr. Hossein-Zadeh is a member of the Optical Society of America (OSA) and the Institute of Physics (IOP).
Anthony F. J. Levi received the Ph.D. degree in physics from Cambridge University, Cambridge, U.K., in 1983.

In mid-1993, he joined the Faculty of the University of Southern California, Los Angeles, after working for ten years with AT\&T Bell Laboratories, Murray Hill, NJ. He invented hot electron spectroscopy, discovered ballistic transport in transistors, created the first microdisk laser, and carried out ground-breaking work in parallel fiber-optic interconnect components in computer and switching systems. His current research interests include the scaling of ultrafast electronic and photonic devices and the system-level integration of advanced opto-electronic technologies, manufacturing at the nanoscale, and the subject of adaptive quantum design. He has authored or coauthored over 200 scientific papers and several book chapters. He authored Applied Quantum Mechanics (Cambridge Univ. Press, 2003). He holds 13 U.S. patents. 